\title{
Wide excision as an optional treatment for large ossifying fibroma: a case report
}

\author{
Hadira, ${ }^{*}$ Adiantoro Seto, Syamsudin Endang
}

\section{Abstract}

Objective: Ossifying fibromas is a benign type of fibro-osseous tumor. These lesions may arise in the maxillary or mandible, but generally have more predilection of the mandible. Women are more often affected.

Methods: A female patient, aged 40 years old, came to the Oral and Maxillofacial Polyclinics at Hasan Sadikin Hospital, complaining of a lump in the upper jaw. The lump effect makes the face asymmetrical, painless and has a hard consistency. We diagnosed this patient with ossifying fibroma. Ossifying fibroma is a kind of lesion that can spread to all directions, so it can resemble a sphere shape. Enucleation and curettage are the common treatment. Larger lesions, which have significant expansion or aggressive growth features, may require resection or wide excision

Results: In this case our treatment ossifying fibroma is wide excision with ferguson webber incision technique and good results.

Conclusion: Ossifying fibromas is a benign type of fibro-osseous tumor. Wide excision is an option for a large size of ossifying fibroma.
Department of Oral and Maxillofacial Surgery, Faculty of Dentistry, Padjadjaran University, General Hospital Dr. Hasan Sadikin, Bandung, Indonesia

*Correspondence to: Hadira, Department of Oral and Maxillofacial Surgery, Faculty of Dentistry, Padjadjaran University, General Hospital Dr. Hasan Sadikin, Bandung, Indonesia hadira.raside@gmail.com

received: 26 May 2018 revised: 2 August 2018 Accepted: 1 October 2018 Available online 1 December 2019

Keywords: Benign, Ferguson webber incision, Lesion, Ossifying fibroma, Wide excision

Cite this Article: Hadira, Seto A, Endang S. 2019. Wide excision as an optional treatment for large ossifying fibroma: a case report. Journal of Dentomaxillofacial Science 4(3): 188-191. D0I: 10.15562/jdmfs.v4i3.756

\section{Introduction}

Ossifying fibromas is a type of benign fibro osseous tumor. These lesions can arise in both jaws, but they have a predilection for the mandible. These lesions are thought to arise from the periodontal ligament. ${ }^{1,2}$ Ossifying fibroma is a "well-circumscribed lesion, which consistens of fibrous tissue, highly cellular in nature with varying amounts of calcified tissue, which gives the appearance as the bone, cementum or both. ${ }^{2}$ Ossifying fibromas occure in second to fourth decade of life with a definite female predilection and maxilla is affected less than mandible, most common site of occurrence is premolar-molar region of the mandible. ${ }^{1,2}$

Clinically, these tumors are round or ovoid, epansile, painless, slow growing mass that displace the roots of adjacent teeth, and it may cause root resorption. Facial asymmetry is noted with larger lesions. $\mathrm{Su}$ et al.70\% cases reported mandible is the most common site of occurrence, with $43 \%$ located in the mandible posterior region, including the ramus area, followed by $22 \%$ located in the maxillary posterior region. . $^{1,2}$

Early lesions may appear completely radiolucent. With maturation, radiopacities appear, and the lesion will eventually become completely radiopaque with a radiolucent rim. Treatment is with enucleation and curettage for most lesions. Larger lesions, which have significant expansion or aggressive growth features, may require resection or wide excision. Surgical technique with extra oral approach can be Transfacial approach to mid-facel lateral rhinotomy and weber-ferguson incision with lynch extension lynch extension. ${ }^{3}$ Recurrence is uncommon, but lifelong surveillance is recommended. ${ }^{1}$ Pathogenesis is still unknown. ${ }^{2,3}$

\section{Case Report}

A female patient, aged 40 years old, came to the Oral and Maxillofacial Polyclinics at Hasan Sadikin Hospital, complaining of a lump in the upper jaw. She said she had for the last 8 years. The lump effect makes the face asymmetrical, the same color as the surrounding tissue. With palpation from extra-oral, we found there was a mass at left mandible, which temperature afebrile, showed hard consistency, no pain, had an palpable edge of mass, appeared localized, tender, had smooth surface, with size measuring $8 \times 6 \times 4 \mathrm{~cm}$ figure 1 . Lymph nodes were not palpable, slow growing mass that displace the roots of adjacent teeth, and it may cause root resorption. No similarity or history of other swelling in her family.

Intraoral examination see figure 2, the appearance of mass causing mobility of the teeth on the left upper jaw, shifting, hard consistency, smooth surface, localized, not easily bleeding, with size measuring $8 \times 6 \times 4 \mathrm{~cm}$ on the left upper jaw and $3 \times 3 \times 3 \mathrm{~cm}$ in the right upper jaw.

This patient had previously performed surgery ektispasi earlier in Bandung area hospital in 2009 


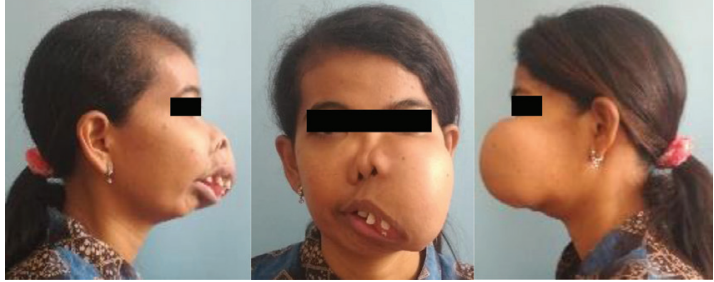

Figure 1 Profile preview before surgery

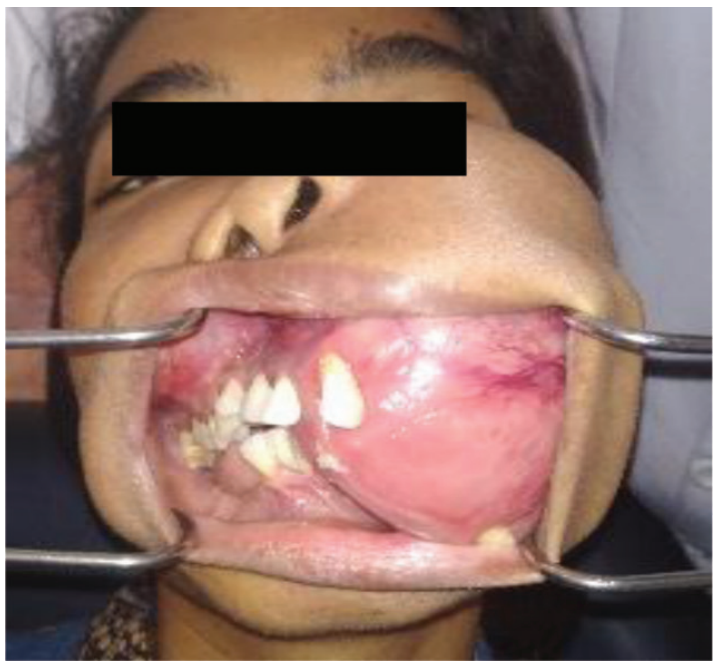

Figure 2 Intraoral view

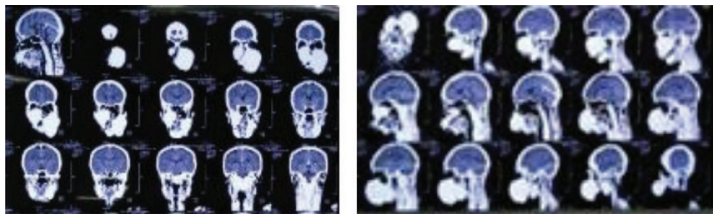

Figure 3 Head CT Scan axial pieces radiograph view
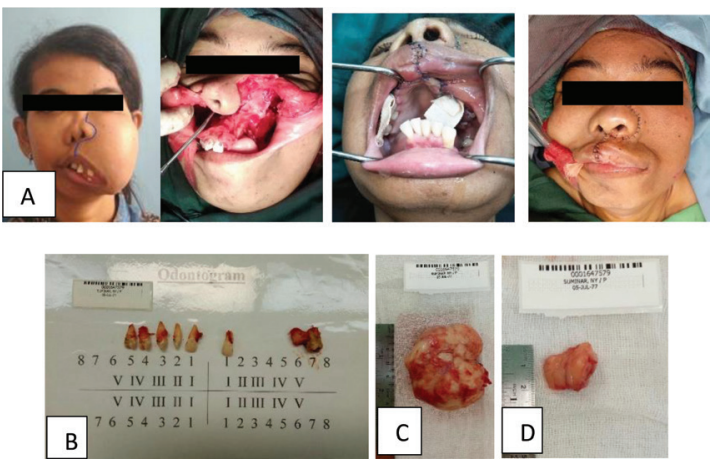

Figure 4 Durante op, A. ferguson webber incision, wide excision at left upper jaw and excision right upper jaw. B. Ekstraksi gigi 15-21,26,27. C \& D Mass of ossifying fibroma

and 2012 with the results of histopatological examination ossifying fibroma. Before surgery is done first taking chest, complete blood examination,
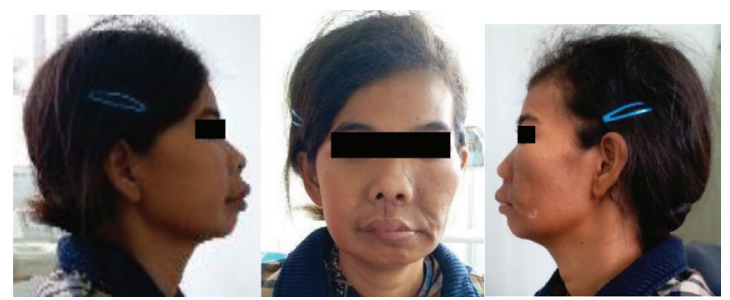

Figure 5 Profile preview fourteen days after surgery

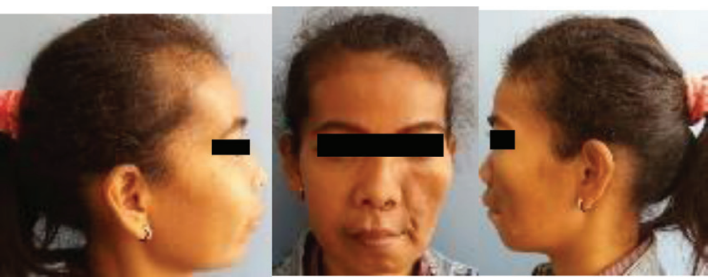

Figure 6 Profile preview 1 moonth after surgery

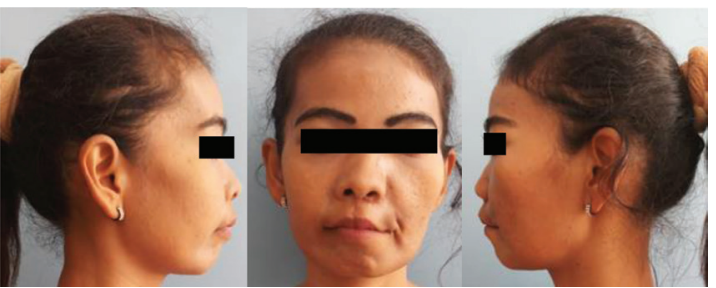

Figure 7 Profile preview two moonth after surgery

chest x-ray, head CT scan and consultation with anesthesia department.

From the complete blood examination and chest $\mathrm{x}$-ray withim normal limit. From were found the head CT scan appears solid mass in the bilateral maxillary that destroys the bilateral maxillary sinus, bone bilateral maxillary and mandible still appear hypergens from the teeth in the bilateral mandible and left maxillary maxillary to osifying fibroma figure 3.

After completing examination, including clinical, complete blood examination, radiograph, and histopathological examinations, we concluded that the patient had a ossifying fibroma at maxilla dextra and sinistra opted wide excision with ferguson webber incision technique was planned to perform under general anesthesia, because the tumor is large.

Surgery was initiated with inci-sional pattern on the left maxilla with methylene blue according to the design ferguson webber incision. Before Incision we do extraction of teeth 15-21, 26, 27. Furthermore, using blade No. 15 from the lateral left nose to nasalis sulcus to the filtrum and ends in the cupid's bow and cauter-ized the incision and blood vessels with ligation of vein and cut the mass form distal tooth 27 mesial tooth 21 and the mass 
from distal tooth 15 until mesial tooth 11. Put in Iodoform kassa, hecting intra oral. Hecting extra oral figure 4. Patients were treated in the nursing room for approximately 5 days with food intake using NGT.

Fourteen days after surgery, and reported that she had no pain and swelling and we did not any signs of infection. we do the appointment iodoform kassa. Patient was then referred to prosthodontic department for prosthesis for printing the preparation of dentures figure 5 .

One moonth day after surgery and reported that she had no pain and swelling and we did not any signs of infection figure 6 . She will plans be tried removable dentures by prosthodontist.

Two moonth day after surgery and reported that she had no pain and swelling and we did not any signs of infection figure 7 .

\section{Discussion}

Ossifying Fibroma is benign, uncommon, monostotic well-defined usually unilocular but occasionally multilocular fibro osseous tumor, arising from the cells of periodontal ligament and is composed of fibrous connective tissue with variable amounts of calcified tissue resembling bone, cementum or both. ${ }^{4}$ Another opinion states that Ossifying fibroma is a fibroma that exhibits osteogenic activity. ${ }^{5}$

Clinically, it presents itself as a nodular lesion, exophytic, pedunculated in the majority of cases, reddish color interspersed with whitish areas or similar coloring to that of the adjacent mucosa. ${ }^{6}$

Histologically, the lesion exhibits a proliferation of fibroblasts associated with the formation of mineralized material which may consist of bone, cementum-like material or dystrophic calcifications. ${ }^{7}$ Radiographically, they may exhibit areas of diffuse radiopaque calcifications, but many lesions do not exhibit this radiographic appearance. ${ }^{8}$

The differential diagnosis of the peripheral ossifying fibroma includes all nodular lesions that occur in the gingiva such as fibroma, giant cells fibroma, pyogenic granuloma and odontogenic neoplasms. ${ }^{6}$

Surgical methode for ossifying fibroma are enucleation, curettage ang recection. In the case we do enucleation with ferguson webber incision, because mass of large size. Surgical technique with extra oral approach are transfacial approach to mid-facel lateral rhinotomy. lateral rhinotomy was first described by michaux in 1848 , but was popularized by moure in 1902 . the classical lateral rhinotomy described by moure lies halfway between the medial canthus and the nasal dorsum extending from the inner margin of the eyebrow down along the nasomaxillary groove curving around the ala to enter into the nose. the incision begins from the columella laterally along the vestibule of the nose and then follows the ala of the nostril and extends along the lateral aspect of the nose to end a centimeter below the inner canthus of the eye. indications access to the nasal cavity, for open rhinoplasties and maxillary antrum. weber-ferguson incision with lynch extension lynch extension. it is an extension on the medial side from the medial canthus to the medial end of the upper eyebrow. indications it is used for extended maxillectomy and access to the medial orbital wall. ${ }^{3,8}$ In this case we do wide excision with ferguson webber incision technique of mass of left maxilla area followed by right maxilla mass excision and result is very significant.

The prognosis of Ossifying Fibroma can be said to be good if at the time of surgery, the tumor mass is lifted entirely. However, if removal of tumor mass is imperfect, then the prognosis is poor. In this case, the mass appointment is done perfectly in the hope that the patient's prognosis is good.

\section{Conclusion}

Ossifying fibromas is a benign type of fibro-osseous tumor. The timely diagnosis and management of this tumor is of utmost importance to limit the progression and to reduce morbidity of the patient. In the present case report, wide excision with ferguson webber incision technique is an option for a large size of ossifying fibroma.

\section{Acknowledgment}

Alhamdulillahi rabil 'alamin, the case reporter expresses his highest gratitude to Allah subhanahu wa ta'ala for blessing, love, opportunity, health, and mercy to complete this case report. This case report entitled "Wide Excision as an optional treatment for large ossifying fibroma: a case report" is submitted as the final requirement in accomplishing spesialist degree at Oral Maxillofacial Surgery, Faculty of Dentistry, Padjadjaran University. In arranging this case report, a lot of people have provided motivation, advice, and support for the case reporter. In this valuable chance, the researcher intended to express his gratitude and appreciation to all of them. First, appreciation goes to her beloved parents, her mother Hj. Naga Yebbo for the endless love, pray, and support, and her father $\mathrm{H}$. Raside for to remind me to keep going and never giving up.

The case report would not have been possible without the help, support and patience of my first advisor, Seto Adiantoro, drg.,Sp.BM(K) for his supervision, advice, and guidance from the very 
early stage of this case report as well as giving me extraordinary experiences throughout the past few years. Then to his second advisor Dr. Endang Syamsudin,drg., Sp.BM(K) who has helped him patiently finishing this case report by giving suggestion, guidance, and correction until the completion of this case report.

Finally, I would like to thank everybody who was important to the successful realization of this case report. This case report is far from perfect, but it is expected that it will be useful not only for the case reporter, but also for the readers. For this reason, constructive thoughtfull suggestion and critics are welcomed.

\section{Conflict of Interest}

The authors report no conflict of interest

\section{References}

1. Hargetty CJ, Laughlin RM. Atlas of operative oral and maxillofacial surgery. Blackwell: John Wiley \& Sons; 2015. p. 409.
2. Vura NG, Gaddipati R, Ramisetti S, et al. Surgical management of ossifying fibroma in maxilla : report of two casess. J Int Oral Health 2015;6: 115-118.

3. Borle MR. Oral and Maxillofacial Surgery. New Delhi: Jaypee Brothers Medical Publishers; 2014. p. 58-61.

4. Bhatt P, Vinod VC, Kaushik A, et all. Ossifying fibroma of the maxilla: a rare case. Int J Maxillofac Imaging 2017;3: 28-31.

5. Kanehira, Marina RP,Valber BM dkk. Surgical Treatment of Peripheral Ossifying Fibroma : a case report. J Oral Diag 2017;3: 1-3.

6. Mishra MB, Bhishen KA, Mishra R. Peripheral ossifying fibroma. J Oral Maxillofac Pathol. 2011;15:65-8.

7. Pal S, Hegde S, Ajila V. The varying clinical presentations of peripheral ossifying fibroma: a report of three cases. Rev Odonto Ciênc. 2012;27:251-5.

8. Pamolango VT, Nurwiadh A, Sylvyana $M$, et al. Hemimandibulectomy of mandibular large odontogenic myxofibroma in adolescence girl and immediate reconstruction with AO plate. J Dentomaxillofac Sci 2016;1: 206-209.

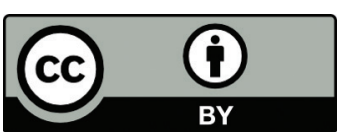

This work is licensed under a Creative Commons Attribution 